\title{
Characterization of Tumor Blood Vasculature Expression of Human Invasive Bladder Cancer by Laser Capture Microdissection and Transcriptional Profiling
}

\author{
Filip Roudnicky, ${ }^{*}$ Cedric Poyet, ${ }^{\dagger}$ Lorenz Buser, ${ }^{\ddagger}$ Karim Saba, ${ }^{\dagger}$ Peter Wild ${ }^{\ddagger}$ Vivianne I. 0tto, ${ }^{*}$ and Michael Detmar*
}

From the Institute of Pharmaceutical Sciences, * Swiss Federal Institute of Technology, Zürich; and the Department of Urology ${ }^{\dagger}$ and the Institute of Pathology and Molecular Pathology, ${ }^{\ddagger}$ University Hospital Zürich, Zürich, Switzerland

Accepted for publication May 27, 2020.

Address correspondence to Michael Detmar, M.D., Institute of Pharmaceutical Sciences Swiss Federal Institute of Technology Zürich, VladimirPrelog-Weg 3, HCI H303 CH8093 Zürich, Switzerland. Email: michael.detmar@pharma. ethz.ch.

\begin{abstract}
Tumor-associated blood vessels differ from normal vessels and play key roles in tumor progression. We aimed to identify biomolecules that are expressed differentially in human bladder cancer-associated blood vessels to find novel biomarkers and mechanisms involved in tumor-associated angiogenesis. The transcriptome of tumor blood vasculature from human invasive bladder carcinoma (I-BLCA) and normal bladder tissue vasculature was compared using differential expression and unsupervised hierarchical clustering analyses. Pathway analysis identified up-regulation of genes involved in the proliferation, cell cycle, angiogenesis, inflammation, and transforming growth factor- $\beta$ signaling in tumor blood vasculature. A common consensus gene expression signature was identified between bladder cancer tumor blood vasculature with tumor blood vasculature of other solid cancers, which correlated with the overall survival of patients with several of the solid cancers investigated in The Cancer Genome Atlas data set. In bladder tumor blood vasculature, the secreted factor angiopoietin-like protein 2 (ANGPTL2), was confirmed to be up-regulated by quantitative RT-PCR and immunohistochemical staining. The upregulation of ANGPTL2 in plasma was also observed in non-invasive bladder carcinoma and I-BLCA. We semiquantitatively analyzed expression of ANGPTL2 in tissue microarrays from I-BLCA and surprisingly found an opposite correlation between staining intensity and progression-free survival. Our results indicate that ANGPTL2 might serve as a potential biomarker to predict progression-free survival in IBLCA. (Am J Pathol 2020, 190: 1960-1970; https://doi.org/10.1016/j.ajpath.2020.05.020)
\end{abstract}

Bladder carcinoma (BLCA) is the second most common malignancy of the urinary tract after prostate cancer ${ }^{1}$ and frequently is noninvasive ${ }^{2}$ (NI-BLCA, pTa). Most NIBLCAs recur (approximately 70\%). but seldom progress to invasive BLCA (I-BLCA; approximately $10 \%$ to $15 \%{ }^{3}$ ). I-BLCA stages pT1 to pT4 (depending on the degree of invasiveness, from pT2, also termed muscle-invasive BLCA) are characterized by high progression rates to a higher stage or nodal-positive disease. ${ }^{4-7}$ The majority of patients will develop metastasis and the 5-year survival is low, even for patients receiving aggressive surgical and systemic treatments. ${ }^{8}$

The extent of angiogenesis has been reported to predict recurrence and progression in NI-BLCA, and a shorter overall survival in I-BLCA, ${ }^{9}$ but the distinct transcriptional profiles of BLCA-associated blood vessels has remained unknown. We used laser capture microdissection (LCM) to isolate tumorassociated blood vasculature and blood vasculature from matched normal adjacent bladder tissue from patients with IBLCA and performed transcriptional profiling using human exon 1.0 sense target arrays. We then compared the I-BLCA tumor blood vasculature signature with tumor blood vasculature from several other solid cancers previously published ${ }^{10-13}$ in The Cancer Genome Atlas (TCGA; https://portal.gdc. cancer.gov) and identified a gene signature that is common to all tumor blood vasculatures and reflects a consensus

\footnotetext{
Supported by the Swiss National Science Foundation grants 147087 166490 and 185392; Advanced European Research Council Grant LYVICAM; Krebsliga Schweiz (M.D.); and Krebsliga Zurich (M.D.).

F.R. and C.P. contributed equally to this work.

Disclosures: None declared.
} 
tumor angiogenesis signature. We next validated the upregulation of angiopoietin-like protein 2 (ANGPTL2) from the LCM material both by quantitative RT-PCR and immunohistochemical staining and correlated ANGPTL2 expression with progression-free survival in I-BLCA. When measured in plasma, the ANGPTL2 levels were significantly higher in patients with NI-BLCA and I-BLCA than in healthy individuals.

\section{Materials and Methods}

\section{Tumor and Normal Tissue Samples}

Fresh tissue samples of I-BLCA and adjacent normal bladder from 6 patients undergoing radical cystectomy (for patient 5 only tumor tissue was received, whereas for patient 6 only normal tissue was received) (Supplemental Table S1 and Figure 1B) were collected at the University Hospital Zurich and were used for LCM. After evaluation by a board-certified pathologist, tissue samples were embedded immediately in Tissue-Tek O.C.T. Compound (Sakura Finetek, Tokyo, Japan) and frozen using liquid nitrogen. The tissue collection was approved by the Ethics Committee (KEK-StV-Nr 02/09) and informed written consent was obtained from each patient. Two tissue microarrays (TMAs) containing I-BLCA from the Institute of Pathology and Molecular Pathology (University Hospital Zurich) were used for evaluation of the prognostic potential of ANGPTL2. All patients from both TMAs underwent radical cystectomy for invasive BLCA. The first TMA consisted of samples from 66 patients and was constructed from a retrospective clinical cohort. The second TMA contained samples from 94 patients and was constructed from a prospective clinical cohort. For the final survival analysis, the clinical and pathologic data of both TMAs were used and consisted of a total of 160 patients. TMAs were approved by the Ethics Committee (StV-Nr. 25/2007 and StV-Nr. 02/09). Clinicopathologic details of the TMAs are provided (Supplemental Table S2).

\section{LCM and Transcriptional Profiling of Blood Vessels from Bladder Tissue}

LCM on frozen sections of I-BLCA and matched normal (tumor-adjacent for four patients, from two patients only tumor or normal-adjacent tissue was received) bladder tissue, RNA isolation, cDNA generation, and microarray hybridization to human 1.0 exon sense target arrays (Affymetrix, Santa Clara, CA) were performed as described. ${ }^{14}$ The microarray data have been deposited in the NCBI GEO database (https://www.ncbi.nlm.nih.gov/geo; accession number GSE41614).

\section{Bioinformatics Analysis of Microarray Data}

Core probe sets from the human exon 1.0 sense target array that map to the same transcript were summarized into a single value using iterPLIER (Thermo Fisher, Carlsbad, CA). ${ }^{15}$ Because exon arrays do not have mismatch probes, background correction was performed on a pool of probes with the same guanine-cytosine content content to generate processed probe set expression intensities. A value of 16 was added to the expression values of each probe set, to stabilize the variance before $\log _{2}$ transformation. Next, the quantile normalization was used to render the distribution of probe intensities for each array the same. This was performed as follows: values on every chip were ranked by their intensity. For every rank, the average over all chips was computed, and the actual values of this rank on every chip then were replaced by this average. Lowly expressed genes, with an expression value between 0 (minimum expression value) and the 20th percentile (low expression value) were removed. Differentially expressed genes were identified using a moderated $t$-test. ${ }^{16}$ Multiple testing correction was performed using the method proposed by Storey and Tibshirani ${ }^{17}$ to generate a false discovery rate-corrected $P$ value (q value). All mentioned analyses were performed in Genespring GX 12 software (Agilent, Santa Clara, CA) and R statistical language. ${ }^{18}$

Unsupervised hierarchical clustering was performed using Spearman rank correlation average linkage using Morpheus (https://software.broadinstitute.org/morpheus, last accessed June 29, 2018). Heatmap analysis was performed with the ClustVis tool ${ }^{19}$ using Canberra clustering distance with average linkage for samples and correlation clustering with average linkage for genes.

\section{Bioinformatics Analysis of Tumor Vasculature of Different Cancer Types}

By using published gene expression studies of tumor vasculature of different cancer types ${ }^{10-13}$ [glioblastoma, ${ }^{10}$ ovarian cancer, ${ }^{11}$ breast cancer ${ }^{13}$ (https://www.ncbi.nlm.nih.gov/geo; accession number GSE41614), renal cell carcinoma ${ }^{12}$ (accession number GSE77199), and colorectal carcinoma ${ }^{12}$ (accession number GSE77199)], and the expression profile of our own study (accession number GSE41614), a $\log _{2}$ fold change gene table of differential expression between tumor and healthy normal tissue vasculature was assembled. Because the gene expression was performed using different microarray platforms with different annotations a number of genes with missing (not available) values were observed. Genes missing from more than three studies were removed; otherwise, the missing values were imputed using the $\mathrm{R}$ package Multivariate Imputation by Chained Equations. The imputed list was analyzed using the Rank Products analysis tool RankProdIt. $^{20,21}$ Survexpress ${ }^{22}$ was used with default conditions. Briefly, all probes (values) of a gene were used to compute an average per sample. The expression values were quantilenormalized to a uniform distribution between 0 and 1 , where 0 stands for the lowest level (not expressed) and 1 for the highest expression. The prognostic index was used to generate 

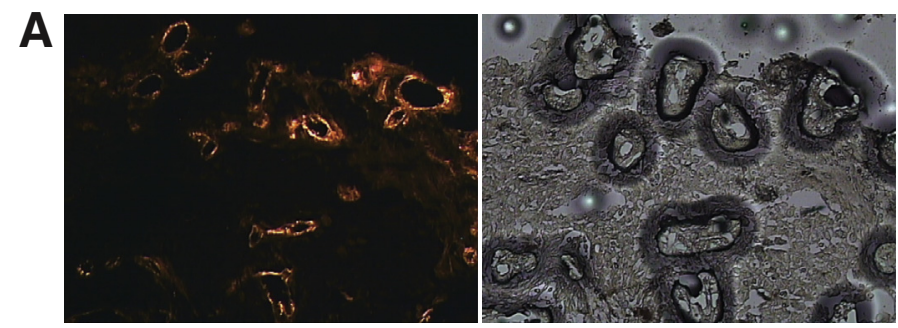

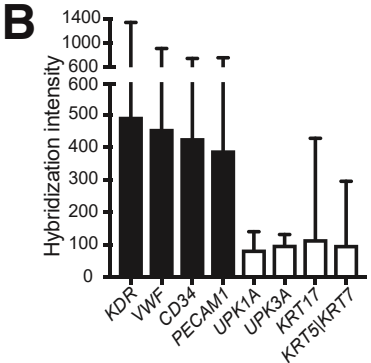

$\mathbf{F}$

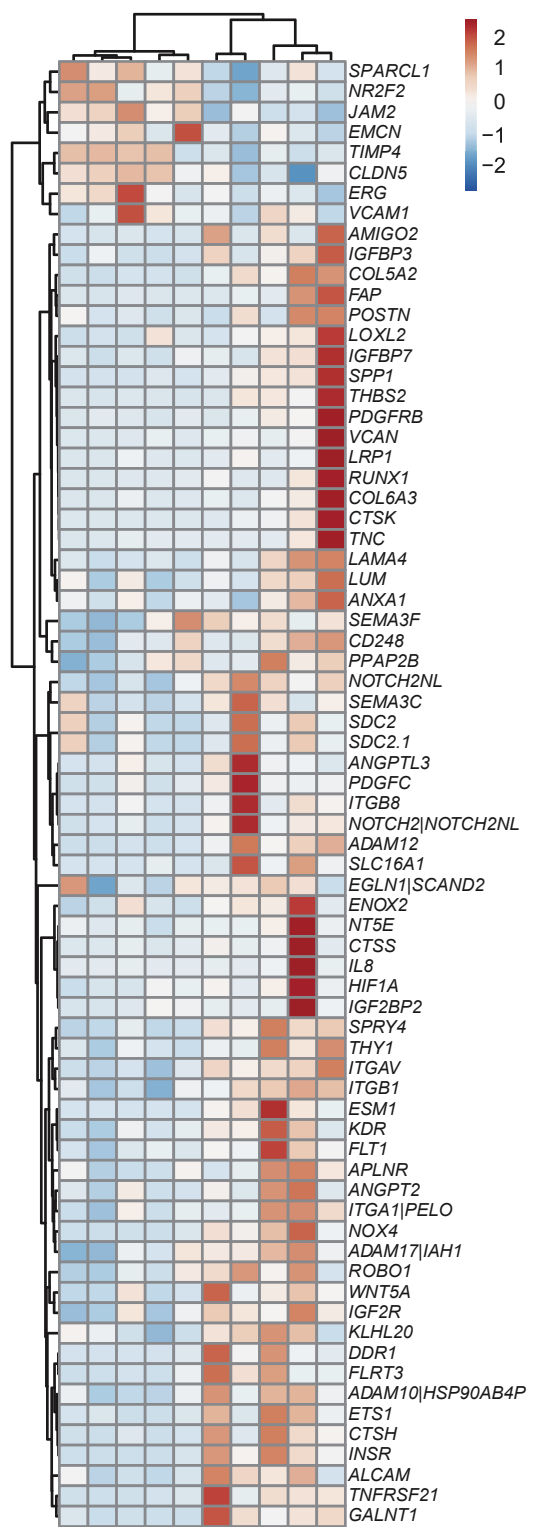

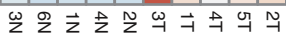
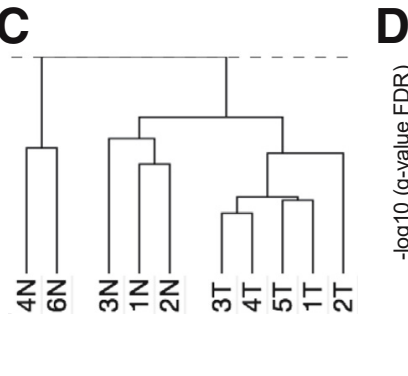

G

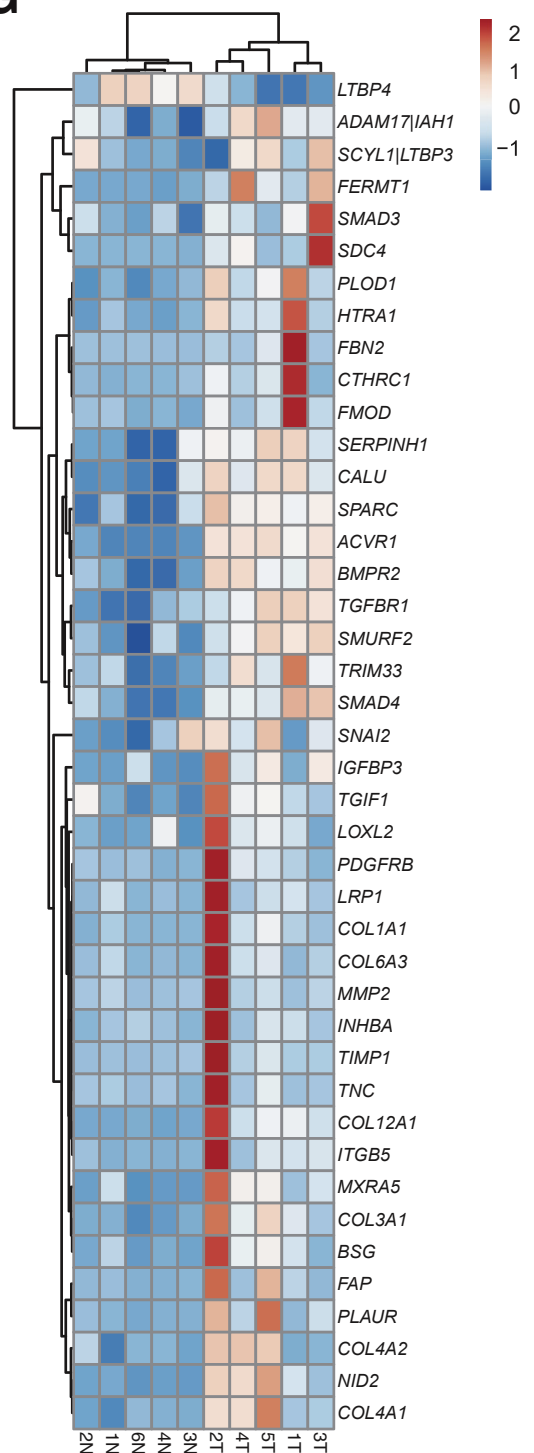

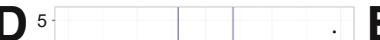
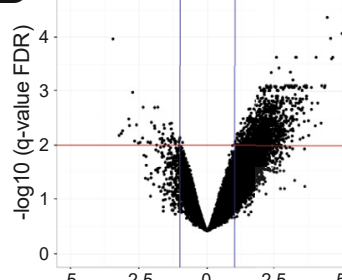

$\begin{array}{lllll}-5 & -2.5 & 0 & 2.5 & 5\end{array}$ log2(Fold change)

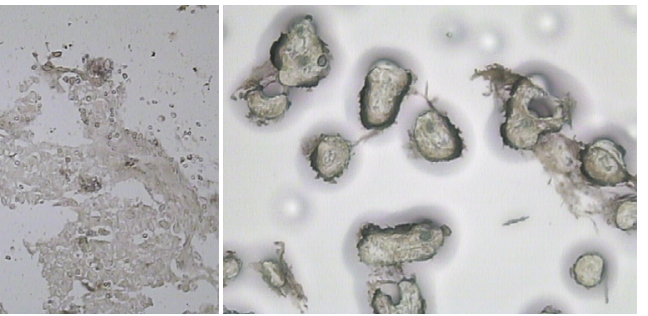

E

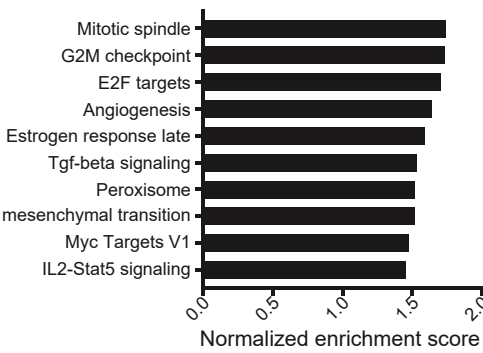

H

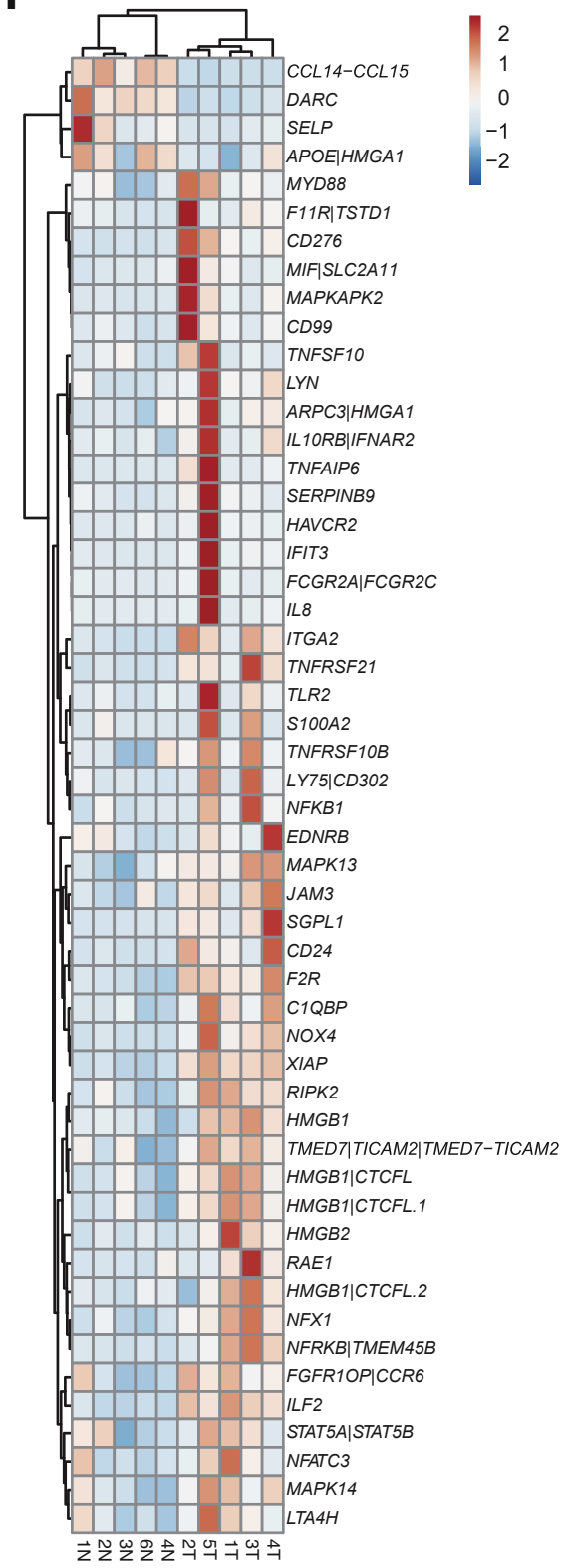


risk groups. It is defined as follows: prognostic index $=\beta 1 x 1+\beta 2 x 2+\ldots+\beta p x p$ where $x l$ is the expression value and the $\beta I$ was obtained from the Cox fitting. Each $\beta I$ can be interpreted as a risk coefficient. Fitting was performed using the $\mathrm{R}$ survival package. The median expression value was used to split the risk group of the prognostic index, generating risk groups of a similar number of samples. The concordance index was calculated using the method developed by Bovelstad and Borgan. ${ }^{23}$ The concordance index estimates the probability that subjects with higher-risk prediction will experience the event after subjects at lower risk. The hazard ratio was calculated using the Cox model using the risk group prediction as the covariate. The log-rank test was used to calculate the $P$ value and the confidence interval.

\section{Immunohistochemical and Immunofluorescence Staining}

Immunohistochemical and immunofluorescence staining were performed as described. ${ }^{14} \mathrm{~A}$ list of the primary and secondary antibodies is provided (Table 1). For the TMA, blood vessels were identified on the basis of their typical morphology by a board-certified pathologist (L.B.), under the supervision of a board-certified and experienced uropathologist (P.W.). The expression of selected proteins on blood vessels was assessed semiquantitatively. For the group with I-BLCA who underwent radical cystectomy, progression-free survival (PFS) and cancer-specific survival (CSS) were evaluated. An increase to a higher tumor stage $^{5,6}$ or the new onset of distant metastasis was considered as progression. CSS was calculated as the time between the date of radical cystectomy and the date of death from bladder carcinoma. Differences between survival estimates were evaluated by the log-rank test and by Cox regression analyses.

\section{Enzyme-Linked Immunosorbent Assay}

Plasma samples from 32 healthy donors and 64 patients with I-BLCA [median age, 72.85 years; age range, 45 to 86 years; male, 50 (78.1\%); female, $14(11.9 \%)]$ and 41 patients with NI-BLCA [median age, 70.5 years; age range, 41 to 87 years; male, 29 (70.7\%); female, $12(29.3 \%)$ ] were analyzed by an ANGPTL2 enzyme-linked immunosorbent assay kit (Immuno-Biological Laboratories, Gunma, Japan) according to the manufacturer's instructions.

\section{Quantitative Real-Time PCR}

Gene expression was quantified using SYBR green quantitative real-time PCR with the AB 7900 HT Fast Real-Time PCR System (Applied Biosystems, Waltham, MA) and the $2^{-\Delta \Delta \mathrm{Ct}}$ method. ${ }^{24}$ Primers were designed spanning the exon-exon junction: ANGPTL2: forward: 5'-GAACCGAGTGCATAAGCAGGA-3', reverse: $5^{\prime}$-GTGAC CCGCGAGTTCATGTT- $3^{\prime}$; and RPLP0: forward: $5^{\prime}$-AGA

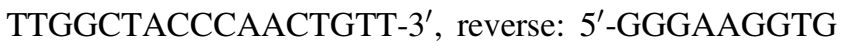
TAATCCGTCTCC- $3^{\prime}$. The expression of ANGPTL2 was normalized to the expression of RPLPO.

\section{Statistical Analysis}

Statistical analyses were performed using Prism version 7.00 (GraphPad Software, Inc., San Diego, CA) and SPSS Statistics version 22 (SPSS; IBM Corp., Armonk, NY). If not stated otherwise, analysis of variance or a two-tailed unpaired $t$-test were used. Survival data were plotted using the KaplanMeier method and analyzed using the log-rank (Mantel-Cox) test or by Cox regression analyses. Differences were considered statistically significant at a $P$ value less than 0.05 .

\section{Results}

\section{Immuno-LCM of Blood Vessels from Bladder Carcinomas and Matched Healthy Tissue and Gene Expression Analysis}

A very fast immunofluorescence staining protocol was optimized using a von Willebrand factor antibody (incubation for only 2 minutes), which enabled visualization of blood vessels and isolation of RNA of high quality (Figure 1A). Microdissection was performed using a farinfrared laser to isolate blood vasculature from the bladder tissue (Figure 1A) of six patients with I-BLCA, followed by transcriptional profiling using human exon 1.0 sense target microarrays. The enrichment of blood vasculature by LCM was confirmed by high expression of the vascular markers CD31 (PECAMI), CD34, VEGFR2 (KDR), and von Willebrand factor $(v W F)$, and low expression of the urothelial and epithelial markers uroplakin1A (UPK1A), uroplakin3A (UPK3A), and keratin 5, 7, and 17 (KRT5, KRT7, KRT17) (Figure 1B), suggesting a several fold enrichment of vasculature tissue over neighboring epithelial tissue.

Figure 1 Immuno-laser capture microdissection (LCM) of blood vessels from bladder carcinomas and matched healthy tissue and gene expression analysis. A: Representative image of a tumor section stained for von Willebrand factor (left panel), the same section before and after LCM (middle panels), and microdissected vessels on the collector cap (right panel). B: Raw microarray expression values (hybridization intensity) of vascular markers (closed bars) and urothelial markers (open bars) in tumor-associated vasculature and healthy-tissue associated vasculature. C: Unsupervised clustering using one minus Spearman rank correlation with average linkage of the microarray gene unfiltered raw intensity expression values. D: Differential expression of tumorassociated vasculature versus healthy-tissue associated vasculature is shown in a Volcano plot. The red line denotes q value false discovery rate (FDR) $<$ 0.01 ; blue lines, $\log _{2}$ fold change $(F C)>1, \log _{2} F C<-1$. E: Gene set enrichment analysis (GSEA) pathway analysis enrichment sorted by normalized enrichment score using Hallmarks MsigDB database of the top 10 up-regulated pathways in tumor-associated vasculature. $\mathbf{F}-\mathbf{H}$ : Heatmap expression analysis using log 2 FC values (red, up-regulated; blue, down-regulated) of genes involved in angiogenesis and hypoxia (F), the transforming growth factor (TGF)- $\beta$ pathway (G), and inflammation $(\mathbf{H})$. Samples were clustered using Canberra clustering with average linkage while the genes were clustered with correlation clustering with average linkage. Data are expressed as median \pm SD (B). 
Table 1 Antibodies Used for Immunohistochemical and Immunofluorescent Studies, Clone Information, and Dilutions or Concentrations Used

\begin{tabular}{|c|c|c|c|c|c|c|c|}
\hline Antibody & Company & $\begin{array}{l}\text { Product } \\
\text { number }\end{array}$ & Immunogen & Host & $\begin{array}{l}\text { Species } \\
\text { reactivity }\end{array}$ & Clone & $\begin{array}{l}\text { Dilution/ } \\
\text { Concentration }\end{array}$ \\
\hline ANGPTL2 & $\begin{array}{l}\text { Sigma-Aldrich, St. } \\
\text { Louis, M0 }\end{array}$ & HPA040933 & $\begin{array}{l}\text { Angiopoietin-like } 2 \\
\text { recombinant } \\
\text { protein epitope } \\
\text { signature tag } \\
\text { (PrEST) }\end{array}$ & Rabbit & Human & Polyclonal & $1: 300$ \\
\hline $\begin{array}{l}\text { von Willebrand } \\
\text { factor }\end{array}$ & $\begin{array}{l}\text { DAK0, Glostrup, } \\
\text { Dennmark }\end{array}$ & A0082 & $\begin{array}{l}\text { von Willebrand } \\
\text { factor isolated } \\
\text { from human } \\
\text { plasma }\end{array}$ & Rabbit & Human & Polyclonal & $15.5 \mu \mathrm{g} / \mathrm{mL}$ \\
\hline $\begin{array}{l}\text { Alexa Fluor } 594 \\
\text { secondary }\end{array}$ & $\begin{array}{l}\text { Thermo Fisher, } \\
\text { Waltham, MA }\end{array}$ & R37119 & $\begin{array}{l}\text { Gamma } \\
\text { immunoglobin }\end{array}$ & Donkey & Rabbit & Polyclonal & $1: 200$ \\
\hline
\end{tabular}

ANGPTL2, angiopoietin like 2; PrEST, protein epitope signature tags.

Unsupervised hierarchical clustering showed that the expression profile of tumor-associated vasculature differed from the vasculature of healthy tissue (Figure 1C). We found 1455 genes to be up-regulated or down-regulated significantly (q value false discovery rate, <0.01) (Figure 1D and Supplemental Table S3). Gene set enrichment analysis based on a gene list sorted by $\log _{2}$ fold change $^{25}$ using the Hallmarks MsigDB database ${ }^{26}$ identified the top 10 enriched pathways in tumor-associated vasculature (Figure 1E and Supplemental Table S4). The top 3 pathways were associated with cellular proliferation (mitotic spindle, G2M checkpoint, and E2F targets), the gene list and heat map expression analysis are shown (Supplemental Figure S1). Pathways with enrichment of genes involved in angiogenesis (Figure 1F), transforming growth factor- $\beta$ signaling and endothelial-mesenchymal transition (Figure 1G), and inflammation (eg, compare IL-2-signal transducer and activator of transcription signaling) (Figure 1, E and $\mathrm{H}$ ) were also detected.

\section{The Signature of Tumor-Associated Bladder}

Vasculature Is Also Found in Tumor-Associated Vessels of Other Solid Cancers and Allows Prediction of Overall Survival

By using several published gene expression studies in tumor vasculature of different solid cancers (glioblastoma, ${ }^{10}$ ovarian, ${ }^{11}$ breast GSE15363 ${ }^{13}$ ), renal cell carcinoma

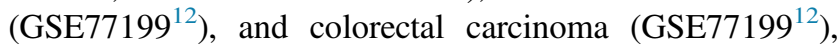
and our study on I-BLCA GSE41614), a $\log _{2}$ fold change differential expression gene list for tumor vasculature compared with matched normal vasculature was assembled (Supplemental Table S5). Because gene expression had been assessed using different microarray platforms, several genes were observed with missing (not available) values (Supplemental Table S5). Genes missing from more than three studies were removed from further analysis; otherwise, the missing values were imputed using the $\mathrm{R}$ package Multivariate Imputation by Chained Equations. The imputed list was analyzed using the Rank Products analysis tool ${ }^{20}$ (Supplemental Table S6). Among the top 10 up-regulated or down-regulated genes, several known angiogenic genes including ESM1, ${ }^{14}$ ANGPT2, ${ }^{27}$ THBS $2,{ }^{28} F^{2} A P,{ }^{29}$ and, respectively, anti-angiogenic molecules including $C H G A,{ }^{30}$ C2orf40 (ECRG4), ${ }^{31}$ and $T T R^{32}$ (Figure 2A) were observed.

By using the ranked list (rank products $\log _{2}$ fold change) of all compared studies that focused on tumor-associated vasculature (Supplemental Table S6), gene set enrichment analysis $^{25}$ using the Hallmark MsigDB database ${ }^{26}$ was performed (Figure 2B and Supplemental Table S7). An enrichment of genes involved in angiogenesis, proliferation, and inflammation similar to the BLCA tumor vasculature (Figure 1E) was observed. Next, genes that showed a rank products up-regulation higher than a $\log _{2}$ fold change of 1 (141 genes) (Supplemental Table S8) were used in Survexpress $^{22}$ to calculate the overall survival prediction in all cancers for which the vasculature was included: bladder carcinoma (Figure 2C), ${ }^{33}$ ovarian cancer,,${ }^{34}$ breast cancer, ${ }^{35}$ glioblastoma ${ }^{36}$ renal cell carcinoma,${ }^{37}$ and colorectal carcinoma. ${ }^{38}$ The gene signature was able to significantly distinguish between high-risk and low-risk cases for overall survival in every analyzed type of cancer as measured by concordance index, hazard ratio, and significance value $(P$ value).

\section{ANGPTL2 Is Up-regulated in Tumor-Associated Blood Vessels of Bladder Cancer and High Expression Correlates Inversely with Survival in Invasive Bladder Cancer}

Gene expression profiling showed up-regulation of ANGPTL2 in tumor-associated blood vessels from six patients with I-BLCA (fold change, 4.97; false discovery rate q value, 0.0098) (Supplemental Table S3). A 200- to 589fold up-regulation of ANGPTL2 mRNA was measured by quantitative RT-PCR in tumor-associated blood vasculature compared with blood vasculature in healthy adjacent tissue (Figure 3A). Immunohistochemical staining showed 


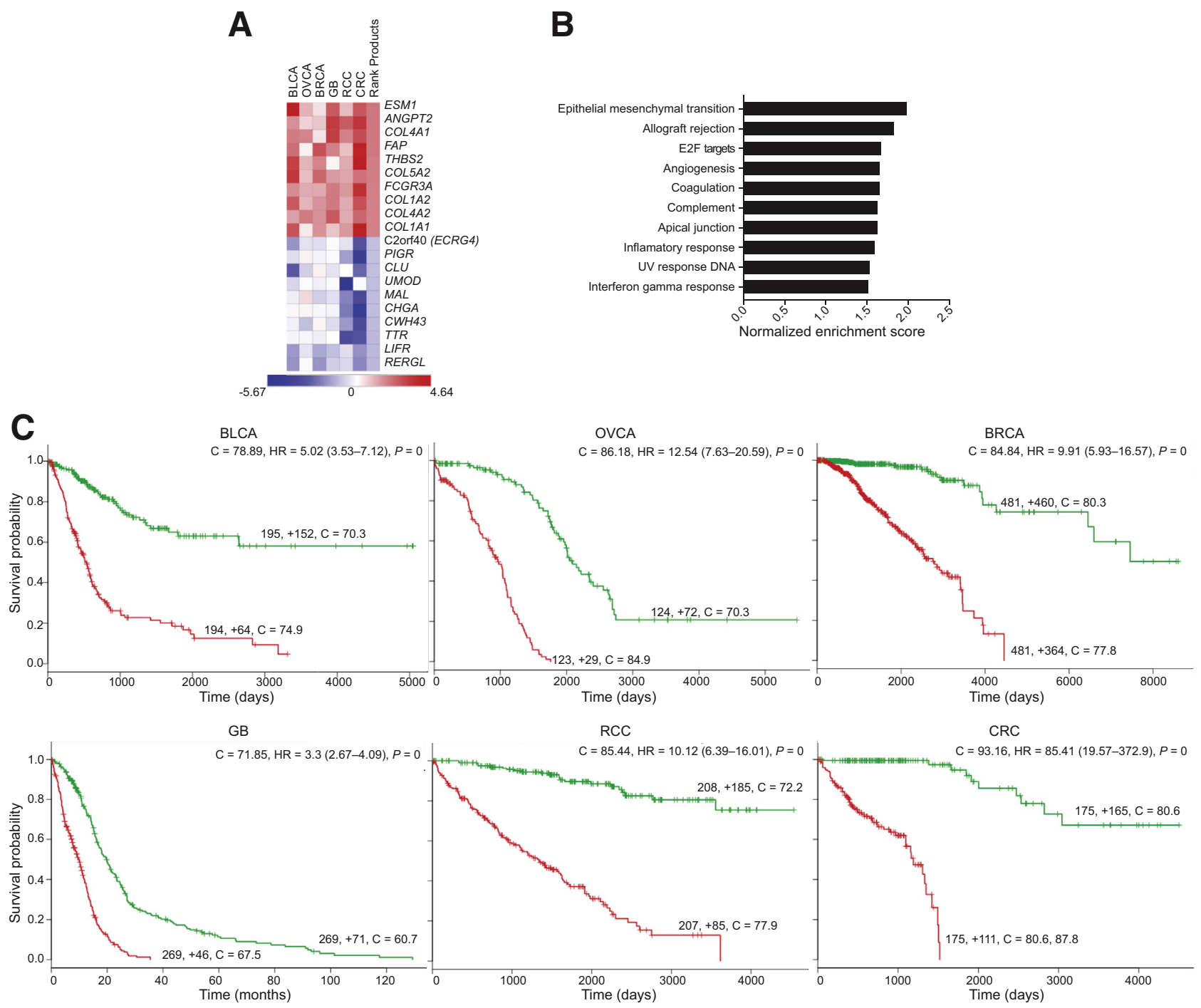

Figure 2 The signature of tumor-associated bladder vasculature is also found in tumor-associated vessels of several other solid cancers and allows prediction of overall survival. A: Heatmap analysis of the top 10 up-regulated (red) and down-regulated (blue) genes from the tumor-associated vasculature of several analyzed cancers [bladder (BLCA), ovarian (OVCA), breast (BRCA), glioblastoma (GB), renal cell carcinoma (RCC), and colorectal carcinoma (CRC)] based on RankProdIt ${ }^{18,19}$ calculations. B: Pathway analysis using gene set enrichment analysis (GSEA) with MsigDB Hallmarks database in the log 2 fold change-sorted tumor-associated vasculature signature of analyzed cancers. C: By using the genes with an up-regulation of expression in tumor-associated vasculature compared with healthy tissue-associated vasculature from all analyzed cancers with a fold-change of more than 2 (141 genes), the association of expression levels with either prolonged (green lines) or short (red lines) overall survival was calculated in The Cancer Genome Atlas (TCGA) data sets of cancers from which the tumor-associated vasculatures were derived. Censoring samples are shown as + symbols. The concordance index (C), hazard ratio with confidence interval, and $P$ value of the log-rank test are shown. The number of individuals, the number of censored patients, and the concordance index of each risk group are shown on each of the curves.

expression in tumor-associated blood vessels (Figure 3B), and some weakly positive vessels in normal bladder tissue (Supplemental Figure S2A) were observed. Urothelial tissue specimens were stained without any evidence of cancer from patients who underwent transurethral resection (Supplemental Figure S2B) and weakly positive vessels were observed. Bladder tissue with evidence of inflammation (Supplemental Figure S2C) was also stained, and only weakly positive vessels were observed. Next, a TMA of I-BLCAs (pT2 to pT40) (Supplemental Table S2) were analyzed and ANGPTL2 expression was assessed semiquantitatively by grading the intensity of the staining
(Figure 3C) as absent (0), weak (1+), or strong (2+). Kaplan-Meier analysis of 152 samples for PFS and 155 for CSS overall survival showed that patients with strong ANGPTL2 expression $\left(n=75, \mathrm{~N}_{\text {events }}=22\right.$ for PFS; $n=$ $76, \mathrm{~N}_{\text {events }}=17$ for CSS) (Figure 3D) had an increased PFS and increased CSS compared with those with no or weak ANGPTL2 expression $\left(n=77, \mathrm{~N}_{\text {events }}=47\right.$ for PFS; $n=$ $79, \mathrm{~N}_{\text {events }}=39$ for CSS; $P<0.001$ ). Furthermore, ANGPTL2 remained an independent predictor for CSS in multivariable Cox regression analyses (adjusted for tumor stage, lymphovascular invasion, and lymph node stage; hazard ratio, $0.46 ; 95 \% \mathrm{CI}, 0.25$ to $0.83 ; P=0.01$ ) 

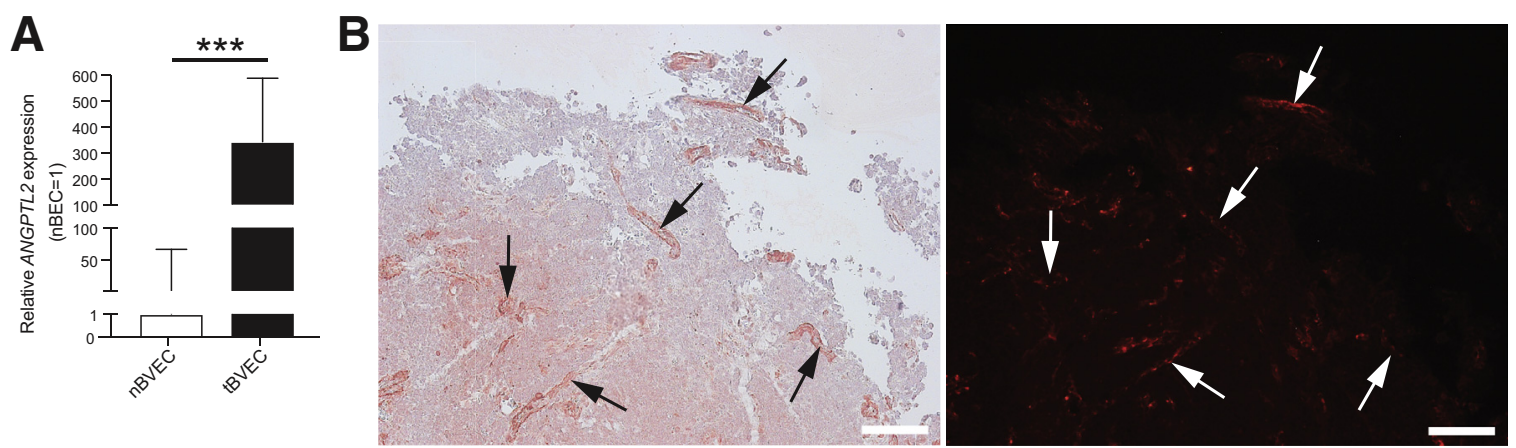

C
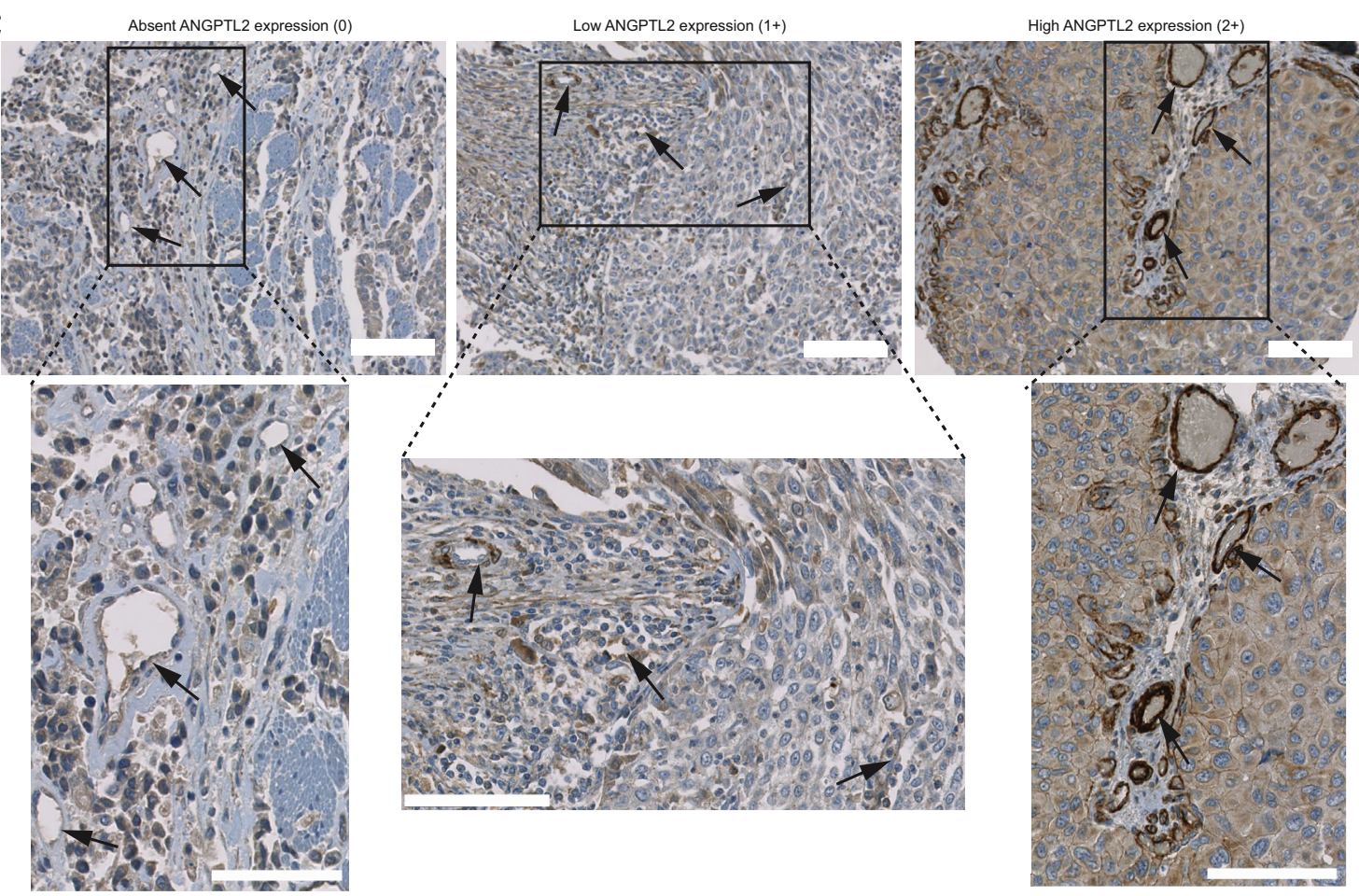

D

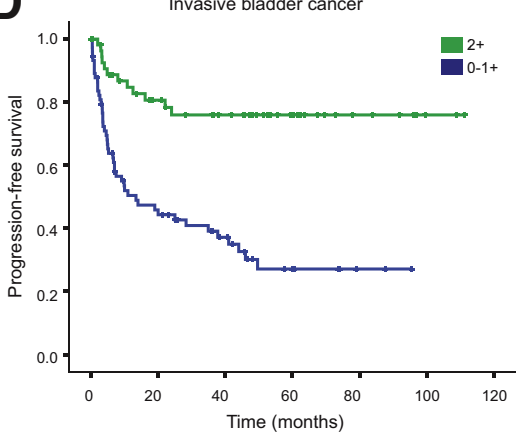

Invasive bladder cancer

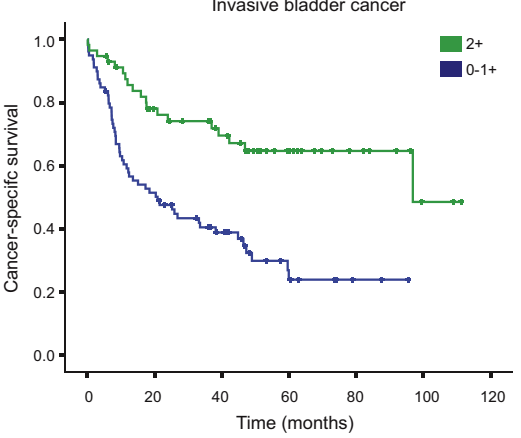

E

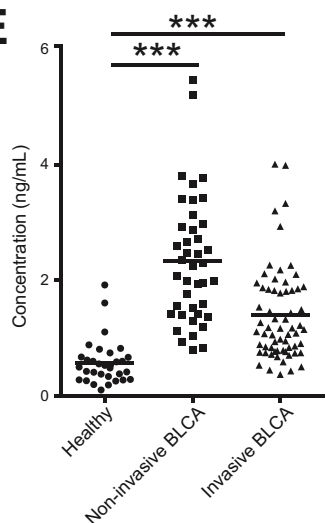

Figure 3 Angiopoietin-like protein 2 (ANGPTL2) is up-regulated in tumor-associated blood vessels of bladder cancer and its high expression inversely correlates with survival in invasive bladder cancer. A: Blood-vasculature endothelial cells (BVECs) were isolated from frozen sections of invasive tumors [tumor blood-vasculature endothelial cells (tBVEC)] and adjacent normal tissue [normal blood-vasculature endothelial cells (nBVEC)] from six bladder cancer patients. ANGPTL2 mRNA was quantified by quantitative RT-PCR and normalized to the mean expression in healthy tissue-associated vasculature. B: Left panel: Paraffin sections of bladder cancer were stained for ANGPTL2 (black arrows). Right panel: To identify the blood vessels, von Willebrand factor (vWF) was detected in the same section (white arrows) by immunofluorescence staining. C: A tissue microarray of 155 human invasive bladder carcinoma (I-BLCA) samples was stained for ANGPTL2. The levels of expression on blood vessels (black arrows) were assessed semiquantitatively by grading the intensity of the staining as absent (0), weak (1+), or strong (2+). D: The Kaplan-Meier curves of progression-free (left panel) and cancer-specific overall survival (right panel) of patients with strong ANGPTL2 (2+) and of those with weak or absent ANGPTL2 expression $(1+, 0)$ are compared. E: ANGPTL2 was measured in the plasma of patients with noninvasive bladder carcinoma (NI-BLCA) $(n=41)$, I-BLCA $(n=$ 64), and in healthy volunteers $(n=32)$ by enzyme-linked immunosorbent assay. Squares and triangles represent patient samples; horizontal lines, mean values. Data are expressed as means \pm SD. ${ }^{* * *} P<0.001$. Scale bars: $100 \mu \mathrm{m}$ (B and $\mathbf{C}$ ). 
(Supplemental Table S9). It was then tested whether increased ANGPTL2 expression in the BLCA vasculature would correlate with increased plasma levels. The mean concentration of ANGPTL2 was significantly higher $(P<0.001, U$-test $)$ in the plasma of patients with noninvasive cancer (all pTa tumors) $(2.33 \mathrm{ng} / \mathrm{mL}$; range, 0.79 to $5.47 \mathrm{ng} / \mathrm{mL} ; n=41)$ and also significantly higher $(P<0.001, U$-test $)$ in the plasma of patients with invasive cancer $(1.4 \mathrm{ng} / \mathrm{mL}$; range, 0.37 to $4.0 \mathrm{ng} / \mathrm{mL} ; n=64)$ than in healthy volunteers $(0.58 \mathrm{ng} / \mathrm{mL}$; range, 0.1 to $1.92 ; n=$ 32) (Figure 3E).

\section{Discussion}

We have established a LCM method for the selected isolation of blood vessels from I-BLCAs and matched normal tissue. Applying this method together with transcriptional profiling using human exon microarrays, we established a comprehensive expression profile of tumor-associated vessels that showed profound differences compared with blood vessels in healthy bladder tissue. Our LCM data cannot exclude that the up-regulation of the identified genes is owing to up-regulation of the genes on smooth muscle cells (eg, pericytes) that with endothelial cells form vasculature. The data from the current study was compared with other studies that studied vasculature from other cancers and used other methods of endothelial cell isolation (eg, fluorescenceactivated cell sorting ${ }^{12}$ ). Based on the comparison of these results with previous studies on isolated tumor-associated vasculature from other solid cancers including glioblastoma, breast, ovarian, colorectal, and renal cell carcinoma, a consensus expression signature for the tumor vasculature was generated. ${ }^{10-13}$ Molecular expression signatures of tumor biopsy specimens, arising mainly from cancer cells, have shown their value in the clinic. ${ }^{39,40}$ Fewer studies have used stromal gene expression ${ }^{41}$ to predict patient response. This study describes the consensus expression signature of tumor vasculature and its predictive power for overall survival. Genes in this signature were shown to be involved in multiple stages of tumor angiogenesis. Vascular endothelial growth factor $\mathrm{A}$ is the main factor activating the process of angiogenesis in response to hypoxia. Indeed, up-regulation of various genes that are known to be up-regulated by hypoxia was found: $C D C P 1$ and $I N S R,{ }^{42}$ and by vascular endothelial growth factor A signaling: ESM1,${ }^{14}$ ANGPT $2,{ }^{10}$ PDGFRB, ADAM12, STC1, and KDR (VEGFR2). One of the first steps in tumor angiogenesis is that the endothelial cells and smooth muscle cells cross the basement membrane and degrade and remodel extracellular matrix. In agreement with this, down-regulation of genes that form the endothelial cell barrier: CLDN5 and JAM2, and up-regulation of matrix metalloproteinase (MMP9, MMP2, and TIMP1), several collagens (COLAA1, COL5A2, COL1A2, COL4A2, COL1A1, COL15A1, COL3A1, and COL12A1), integrins (ITGA1, ITGB2, ITGB1, and ITGAV), and laminins (LAMA4 and $L A M B 1$ ) was observed. We also identified up-regulation of genes involved in the proliferation of endothelial cells: KIF11, CENPF, FOLH1, DLGAP5, and TOP2A.

The prevalence and importance of transforming growth factor- $\beta$ and endothelial-mesenchymal transition in tumor vasculature have been debated. ${ }^{43,44}$ Up-regulation of the majority of genes associated with endothelial-mesenchymal transition was noted: SPP1, TGFBI, ACVR1, FN1, CLDN1, ASPM, SMAD5, SMAD2, COL1A2, COLAA1, and COLAA2. ${ }^{10}$ Transforming growth factor- $\beta$ is able not only to induce endothelial-mesenchymal transition but also has immunosuppressive effects and causes endothelial anergy, an inability to respond to inflammation and attract immune cells. ${ }^{45,46}$ An up-regulation of the endothelin B receptor, ${ }^{47}$ which suppresses adhesion of $\mathrm{T}$ cells to endothelial cells and down-regulation of Duffy antigen receptor for chemokines (DARC), a chemokine decoy receptor was noted. ${ }^{30}$ Furthermore, an increased expression of proinflammatory mediators in tumor-associated vasculature was detected, including these factors that have been shown previously to have a role in cancer progression: $H M G B 1,{ }^{48} H M G B 2,{ }^{49}$ NOX $4{ }^{50}{ }^{\text {TNFRSF } 21,{ }^{51} \text { MAPK13, }}{ }^{52}$ MAPK $14,{ }^{53}$ NFATC $3{ }^{54}$ and STAT5. ${ }^{55}$ Finally, up-regulation of the CIQBP,${ }^{56}{\text { CXCR } 4,{ }^{57} \text { VCAN, }}^{58}$ and ANGPTL $2{ }^{59,60}$ genes in tumor blood vasculature that have been shown to participate in tumor progression and metastasis in mouse cancer models were found. In conclusion, the identified consensus gene expression profile characterizes a proliferative, immunosuppressive, but inflamed vasculature that potentially undergoes endothelial to mesenchymal transition. This consensus signature will be of use as a comprehensive resource for vascular biologists and oncologists.

From the identified signature we focused on ANGPTL2, which was highly expressed in tumor blood vasculature from bladder cancer. ANGPTLs are structurally similar to angiopoietins, but they do not bind to tyrosine kinase with immunoglobulin-like and EGF-like domains 1 (Tie1) or Tie2 receptors. ${ }^{61}$ Previously, angiopoietin-2 expression was found to correlate with increased histologic grade and histologic stage, poor prognosis, ${ }^{62}$ and recurrence ${ }^{63}$ of human bladder cancer. Because ANGPTL2 it was structurally similar to angiopoietin-2 and a secreted factor, we explored whether it might serve as a suitable marker of progression and survival in patients with bladder cancer.

Interestingly, in I-BLCA, an inverse correlation as patients with lower expression of ANGPTL2 had the shorter PFS and CSS was observed. ANGTPL2 vessel staining remained an independent predictor for CSS, even when adjusted for known pathologic prognostic factors such as tumor stage or lymphovascular invasion. ANGPTL2 is a secreted protein; it is accessible as a biomarker in blood fluids. ANGPTL2 amounts in plasma were measured, and an increase in patients with I-BLCA and NI-BLCA were identified when compared with healthy individuals. It is difficult to understand why high ANGPTL2 expression by tumor-associated blood vessels was associated with 
prolonged PFS in patients with I-BLCA. Most likely, ANGPTL2 does not play a direct role in tumor progression. Its expression in tumor-associated blood vessels may be increased in the context of different disease processes. One such disease process could be inflammation. ${ }^{64}$ ANGPTL2 is known to activate macrophages, promote proinflammatory macrophage phenotypes, and stimulate inflammatory cytokine secretion by macrophages. ${ }^{65}$ Inflammation in bladder cancer that is associated with angiogenesis has shown correlation with a good prognosis in bladder cancer. ${ }^{66}$ Thus, increased ANGPTL2 expression in the context of an inflammation may hinder disease progression in noninvasive BLCA. On the other hand, in a chemically induced mouse model of skin squamous cell carcinoma, ANGPTL2 expression correlated with the frequency of carcinogenesis, $^{59}$ and in a xenograft mouse model, tumor cell-derived ANGPTL2 accelerated metastasis and induced angiogenesis, ${ }^{60}$ suggesting that there was a direct association of high ANGPTL2 expression with increased carcinogenesis. ${ }^{59,60}$ Thus, there may be other disease processes associated with high ANGPTL2 expression that are responsible for the reduction of PFS in patients with I-BLCA. Future studies are needed to elucidate the functions of ANGPTL2 expression by endothelial cells under different pathologic conditions.

In summary, this study describes the gene expression profile of tumor blood vasculature in I-BLCA for the first time. It also shows a consensus expression profile that the tumor blood vasculature of several solid cancers have in common. The identified transcripts may be evaluated for their suitability as tumor biomarkers or novel therapeutic targets.

\section{Acknowledgments}

We thank Jeannette Scholl and Peter Camenzind for technical assistance, and Lothar C. Dieterich and Ronald J. Buckanovich for sharing raw microarray expression files.

\section{Supplemental Data}

Supplemental material for this article can be found at http://doi.org/10.1016/j.ajpath.2020.05.020.

\section{References}

1. Jemal A, Bray F, Center MM, Ferlay J, Ward E, Forman D: Global cancer statistics. CA Cancer J Clin 2011, 61:69-90

2. Castillo-Martin M, Domingo-Domenech J, Karni-Schmidt O, Matos T, Cordon-Cardo C: Molecular pathways of urothelial development and bladder tumorigenesis. Urol Oncol 2011, 28:401-408

3. Roupret M, Babjuk M, Comperat E, Zigeuner R, Sylvester RJ, Burger M, Cowan NC, Gontero P, Van Rhijn BWG, Mostafid AH, Palou J, Shariat SF: European Association of Urology Guidelines on upper urinary tract urothelial carcinoma: 2017 update. Eur Urol 2018, $73: 111-122$
4. Hautmann RE, de Petriconi RC, Pfeiffer C, Volkmer BG: Radical cystectomy for urothelial carcinoma of the bladder without neoadjuvant or adjuvant therapy: long-term results in 1100 patients. Eur Urol 2012, 61:1039-1047

5. Sobin LH: The WHO histological classification of urinary bladder tumours. Urol Res 1978, 6:193-195

6. Montironi R, Lopez-Beltran A: The 2004 WHO classification of bladder tumors: a summary and commentary. Int J Surg Pathol 2005, 13:143-153

7. Mostofi FK, Sobin LH, Torloni H; World Health Organization: Histological typing of urinary bladder tumours/F. K. Mostofi, in collaboration with L. H. Sobin, H. Torloni and pathologists in fourteen countries. Geneva, World Health Organization, 1973

8. Witjes JA, Compérat E, Cowan NC, De Santis M, Gakis G, Lebret T, Ribal MJ, Van der Heijden AG, Sherif A: EAU guidelines on muscleinvasive and metastatic bladder cancer: summary of the 2013 guidelines. Eur Urol 2014, 65:778-792

9. Streeter EH, Harris AL: Angiogenesis in bladder cancer-prognostic marker and target for future therapy. Surg Oncol 2002, 11:85-100

10. Dieterich LC, Mellberg S, Langenkamp E, Zhang L, Zieba A, Salomaki H, Teichert M, Huang H, Edqvist PH, Kraus T, Augustin HG, Olofsson T, Larsson E, Soderberg O, Molema G, Ponten F, Georgii-Hemming P, Alafuzoff I, Dimberg A: Transcriptional profiling of human glioblastoma vessels indicates a key role of VEGF-A and TGFbeta2 in vascular abnormalization. J Pathol 2012, 228:378-390

11. Buckanovich RJ, Sasaroli D, O'Brien-Jenkins A, Botbyl J, Hammond R, Katsaros D, Sandaltzopoulos R, Liotta LA, Gimotty PA, Coukos G: Tumor vascular proteins as biomarkers in ovarian cancer. J Clin Oncol 2007, 25:852-861

12. Wragg JW, Finnity JP, Anderson JA, Ferguson HJ, Porfiri E, Bhatt RI, Murray PG, Heath VL, Bicknell R: MCAM and LAMA4 are highly enriched in tumor blood vessels of renal cell carcinoma and predict patient outcome. Cancer Res 2016, 76:2314-2326

13. Pepin F, Bertos N, Laferriere J, Sadekova S, Souleimanova M, Zhao H, Finak G, Meterissian S, Hallett MT, Park M: Gene-expression profiling of microdissected breast cancer microvasculature identifies distinct tumor vascular subtypes. Breast Cancer Res 2012, 14:R120

14. Roudnicky F, Poyet C, Wild P, Krampitz S, Negrini F, Huggenberger R, Rogler A, Stohr R, Hartmann A, Provenzano M, Otto VI, Detmar M: Endocan is upregulated on tumor vessels in invasive bladder cancer where it mediates VEGF-A-induced angiogenesis. Cancer Res 2013, 73:1097-1106

15. Affymetrix: Gene Signal Estimates from Exon Arrays. Santa Clara, CA, Affymetrix, 2005

16. Smyth GK: Linear models and empirical Bayes methods for assessing differential expression in microarray experiments. Stat Appl Genet Mol Biol 2004, 3. Article3

17. Storey JD, Tibshirani R: Statistical significance for genomewide studies. Proc Natl Acad Sci U S A 2003, 100:9440-9445

18. Ihaka R, Gentleman R: R: a language for data analysis and graphics Journal of Computational and Graphical Statistics 1996, 5:299-314

19. Metsalu T, Vilo J: ClustVis: a web tool for visualizing clustering of multivariate data using principal component analysis and heatmap. Nucleic Acids Res 2015, 43:W566-W570

20. Laing E, Smith CP: RankProdIt: a web-interactive rank products analysis tool. BMC Res Notes 2010, 3:221

21. Hong F, Breitling R, McEntee CW, Wittner BS, Nemhauser JL, Chory J: RankProd: a Bioconductor package for detecting differentially expressed genes in meta-analysis. Bioinformatics 2006, 22: $2825-2827$

22. Aguirre-Gamboa R, Gomez-Rueda H, Martinez-Ledesma E, Martinez-Torteya A, Chacolla-Huaringa R, Rodriguez-Barrientos A, Tamez-Pena JG, Trevino V: SurvExpress: an online biomarker validation tool and database for cancer gene expression data using survival analysis. PLoS One 2013, 8:e74250 
23. Bovelstad HM, Borgan O: Assessment of evaluation criteria for survival prediction from genomic data. Biom J 2011, 53:202-216

24. Schmittgen TD, Livak KJ: Analyzing real-time PCR data by the comparative C(T) method. Nat Protoc 2008, 3:1101-1108

25. Subramanian A, Tamayo P, Mootha VK, Mukherjee S, Ebert BL, Gillette MA, Paulovich A, Pomeroy SL, Golub TR, Lander ES, Mesirov JP: Gene set enrichment analysis: a knowledge-based approach for interpreting genome-wide expression profiles. Proc Natl Acad Sci U S A 2005, 102:15545-15550

26. Liberzon A, Birger C, Thorvaldsdottir H, Ghandi M, Mesirov JP, Tamayo P: The Molecular Signatures Database (MSigDB) hallmark gene set collection. Cell Syst 2015, 1:417-425

27. Huang H, Lai J-Y, Do J, Liu D, Li L, Del Rosario J, Doppalapudi VR, Pirie-Shepherd S, Levin N, Bradshaw C: Specifically targeting angiopoietin-2 inhibits angiogenesis, Tie2-expressing monocyte infiltration, and tumor growth. Nat Commun 2011, 17:1001-1011

28. Roudnicky F, Yoon SY, Poghosyan S, Schwager S, Poyet C, Vella G, Bachmann SB, Karaman S, Shin JW, Otto VI, Detmar M: Alternative transcription of a shorter, non-anti-angiogenic thrombospondin-2 variant in cancer-associated blood vessels. Oncogene 2018, 37:2573-2585

29. Choi S-H, Kim AR, Nam J-K, Kim J-M, Kim J-Y, Seo HR, Lee H-J, Cho J, Lee Y-J: Tumour-vasculature development via endothelial-tomesenchymal transition after radiotherapy controls CD44v6+ cancer cell and macrophage polarization. Nat Commun 2018, 9:5108

30. Curnis F, Dallatomasina A, Bianco M, Gasparri A, Sacchi A, Colombo B, Fiocchi M, Perani L, Venturini M, Tacchetti C, Sen S, Borges R, Dondossola E, Esposito A, Mahata SK, Corti A: Regulation of tumor growth by circulating full-length chromogranin A. Oncotarget 2016, 7:72716-72732

31. Mao L, Li X, Gong S, Yuan H, Jiang Y, Huang W, Sun X, Dang X: Serum exosomes contain ECRG4 mRNA that suppresses tumor growth via inhibition of genes involved in inflammation, cell proliferation, and angiogenesis. Cancer Gene Ther 2018, 25:248-259

32. Shao J, Yin Y, Yin X, Ji L, Xin Y, Zou J, Yao Y: Transthyretin exerts pro-apoptotic effects in human retinal microvascular endothelial cells through a GRP78-dependent pathway in diabetic retinopathy. Cell Physiol Biochem 2017, 43:788-800

33. Cancer Genome Atlas Research Network: Comprehensive molecular characterization of urothelial bladder carcinoma. Nature 2014, 507: 315-322

34. Cancer Genome Atlas Research Network: Integrated genomic analyses of ovarian carcinoma. Nature 2011, 474:609-615

35. Cancer Genome Atlas Research Network: Comprehensive molecular portraits of human breast tumours. Nature 2012, 490:61-70

36. Cancer Genome Atlas Research Network: Comprehensive genomic characterization defines human glioblastoma genes and core pathways. Nature 2008, 455:1061-1068

37. Ricketts CJ, De Cubas AA, Fan H, Smith CC, Lang M, Reznik E, et al: The Cancer Genome Atlas comprehensive molecular characterization of renal cell carcinoma. Cell Rep 2018, 23:3698

38. Cancer Genome Atlas Research Network: Comprehensive molecular characterization of human colon and rectal cancer. Nature 2012, 487: $330-337$

39. van de Vijver MJ, He YD, van 't Veer LJ, Dai H, Hart AAM, Voskuil DW, Schreiber GJ, Peterse JL, Roberts C, Marton MJ, Parrish M, Atsma D, Witteveen A, Glas A, Delahaye L, van der Velde T, Bartelink H, Rodenhuis S, Rutgers ET, Friend SH, Bernards R: A gene-expression signature as a predictor of survival in breast cancer. N Engl J Med 2002, 347:1999-2009

40. Ludwig JA, Weinstein JN: Biomarkers in cancer staging, prognosis and treatment selection. Nat Rev Cancer 2005, 5:845

41. Finak G, Bertos N, Pepin F, Sadekova S, Souleimanova M, Zhao H, Chen H, Omeroglu G, Meterissian S, Omeroglu A, Hallett M, Park M: Stromal gene expression predicts clinical outcome in breast cancer. Nat Med 2008, 14:518

42. Roudnicky F, Dieterich LC, Poyet C, Buser L, Wild P, Tang D, Camenzind P, Ho CH, Otto VI, Detmar M: High expression of insulin receptor on tumour-associated blood vessels in invasive bladder cancer predicts poor overall and progression-free survival. J Pathol 2017, 242:193-205

43. Man S, Sanchez Duffhues G, ten Dijke P, Baker D: The therapeutic potential of targeting the endothelial-to-mesenchymal transition. Angiogenesis 2019, 22:3-13

44. Cho JG, Lee A, Chang W, Lee MS, Kim J: Endothelial to mesenchymal transition represents a key link in the interaction between inflammation and endothelial dysfunction. Front Immunol 2018, 9:294

45. Klein D: The tumor vascular endothelium as decision maker in cancer therapy. Front Oncol 2018, 8:367

46. Schaaf MB, Garg AD, Agostinis P: Defining the role of the tumor vasculature in antitumor immunity and immunotherapy. Cell Death Dis 2018, 9:115

47. Buckanovich RJ, Facciabene A, Kim S, Benencia F, Sasaroli D, Balint K, Katsaros D, O’Brien-Jenkins A, Gimotty PA, Coukos G: Endothelin B receptor mediates the endothelial barrier to T cell homing to tumors and disables immune therapy. Nat Med 2008, 14:28-36

48. Tang D, Kang R, Zeh HJ 3rd, Lotze MT: High-mobility group box 1 and cancer. Biochim Biophys Acta 2010, 1799:131-140

49. Wang $\mathrm{W}$, Jiang $\mathrm{H}$, Zhu $\mathrm{H}$, Zhang $\mathrm{H}$, Gong J, Zhang L, Ding Q: Overexpression of high mobility group box 1 and 2 is associated with the progression and angiogenesis of human bladder carcinoma. Oncol Lett 2013, 5:884-888

50. Chen C, Li L, Zhou HJ, Min W: The role of NOX4 and TRX2 in angiogenesis and their potential cross-talk. Antioxidants (Basel) 2017, 6:42

51. Strilic B, Yang L, Albarran-Juarez J, Wachsmuth L, Han K, Muller UC, Pasparakis M, Offermanns S: Tumour-cell-induced endothelial cell necroptosis via death receptor 6 promotes metastasis. Nature 2016, 536:215-218

52. Wada M, Canals D, Adada M, Coant N, Salama MF, Helke KL, Arthur JS, Shroyer KR, Kitatani K, Obeid LM, Hannun YA: P38 delta MAPK promotes breast cancer progression and lung metastasis by enhancing cell proliferation and cell detachment. Oncogene 2017, 36:6649-6657

53. Igea A, Nebreda AR: The stress kinase p38alpha as a target for cancer therapy. Cancer Res 2015, 75:3997-4002

54. Pan MG, Xiong Y, Chen F: NFAT gene family in inflammation and cancer. Curr Mol Med 2013, 13:543-554

55. Koppikar P, Lui VW, Man D, Xi S, Chai RL, Nelson E, Tobey AB, Grandis JR: Constitutive activation of signal transducer and activator of transcription 5 contributes to tumor growth, epithelialmesenchymal transition, and resistance to epidermal growth factor receptor targeting. Clin Cancer Res 2008, 14:7682-7690

56. Bulla R, Tripodo C, Rami D, Ling GS, Agostinis C, Guarnotta C, Zorzet S, Durigutto P, Botto M, Tedesco F: C1q acts in the tumour microenvironment as a cancer-promoting factor independently of complement activation. Nat Commun 2016, 7:10346

57. Chatterjee S, Behnam Azad B, Nimmagadda S: The intricate role of CXCR4 in cancer. Adv Cancer Res 2014, 124:31-82

58. Asano K, Nelson CM, Nandadasa S, Aramaki-Hattori N, Lindner DJ, Alban T, Inagaki J, Ohtsuki T, Oohashi T, Apte SS, Hirohata S: Stromal versican regulates tumor growth by promoting angiogenesis. Sci Rep 2017, 7:17225

59. Aoi J, Endo M, Kadomatsu T, Miyata K, Nakano M, Horiguchi H, Ogata A, Odagiri H, Yano M, Araki K, Jinnin M, Ito T, Hirakawa S, Ihn H, Oike Y: Angiopoietin-like protein 2 is an important facilitator of inflammatory carcinogenesis and metastasis. Cancer Res 2011, 71: $7502-7512$

60. Endo M, Nakano M, Kadomatsu T, Fukuhara S, Kuroda H, Mikami S, Hato T, Aoi J, Horiguchi H, Miyata K, Odagiri H, Masuda T, Harada M, Horio H, Hishima T, Nomori H, Ito T, Yamamoto Y, Minami T, Okada S, Takahashi T, Mochizuki N, Iwase $\mathrm{H}$, Oike $\mathrm{Y}$ : Tumor cell-derived angiopoietin-like protein ANGPTL2 is a critical driver of metastasis. Cancer Res 2012, 72: $1784-1794$ 
61. Hato T, Tabata M, Oike Y: The role of angiopoietin-like proteins in angiogenesis and metabolism. Trends Cardiovasc Med 2008, 18:6-14

62. Oka N, Yamamoto Y, Takahashi M, Nishitani M, Kanayama HO, Kagawa S: Expression of angiopoietin-1 and -2, and its clinical significance in human bladder cancer. BJU Int 2005, 95:660-663

63. Szarvas T, Jager T, Totsch M, vom Dorp F, Kempkensteffen C, Kovalszky I, Romics I, Ergun S, Rubben H: Angiogenic switch of angiopoietins-Tie2 system and its prognostic value in bladder cancer. Clin Cancer Res 2008, 14:8253-8262

64. Kadomatsu T, Endo M, Miyata K, Oike Y: Diverse roles of ANGPTL2 in physiology and pathophysiology. Trends Endocrinol Metab 2014, 25:245-254
65. Horio E, Kadomatsu T, Miyata K, Arai Y, Hosokawa K, Doi Y, Ninomiya T, Horiguchi H, Endo M, Tabata M, Tazume H, Tian Z, Takahashi O, Terada K, Takeya M, Hao H, Hirose N, Minami T, Suda T, Kiyohara Y, Ogawa H, Kaikita K, Oike Y: Role of endothelial cell-derived angptl2 in vascular inflammation leading to endothelial dysfunction and atherosclerosis progression. Arterioscler Thromb Vasc Biol 2014, 34:790-800

66. Offersen BV, Knap MM, Marcussen N, Horsman MR, HamiltonDutoit S, Overgaard J: Intense inflammation in bladder carcinoma is associated with angiogenesis and indicates good prognosis. $\mathrm{Br} \mathrm{J}$ Cancer 2002, 87:1422-1430 